\title{
Geodesics and Curvature of a Group of Diffeomorphisms and Motion of an Ideal Fluid
}

AUTHOR(S):

Nakamura, F.; Hattori, Y.; Kambe, T.

\section{CITATION:}

Nakamura, F....[et al]. Geodesics and Curvature of a Group of Diffeomorphisms and Motion of an Ideal Fluid. 数理解析研究所講究録 1991, 769: 62-73

ISSUE DATE:

1991-11

URL:

http://hdl.handle.net/2433/82349

RIGHT: 


\title{
Geodesics and Curvature of a Group of Diffeomorphisms and Motion of an Ideal Fluid
}

\author{
F. Nakamura, Y. Hattori and T. Kambe \\ Department of Physics, University of Tokyo, Bunkyo-ku, Tokyo 113, Japan
}

\begin{abstract}
Motion of an ideal fluid is represented as geodesics on the group of all volume preserving diffeomorphisms. Explicit form of the geodesic equation is presented for the fluid flow on a three-torus. Riemannian connection, cornmutator and curvature tensor are given explicitly and applied to a couple of simple flows with Beltrami property. It is found that the curvature is non-positive for the section of two $A B C$ flows with different values of the constants (A, B and C). The present study is an extension of the Arnold's results in two dimensional case to three dimensional fluid motions.
\end{abstract}


We are concerned with a method to connect the problem of hydrodynamics of an ideal (incompressible and inviscid) fluid with a problem of finding geodesics on the group of all volume preserving diffeomorphisms. The fact that this group is the appropriate configuration space for the hydrodynamics of an ideal fluid was first remarked by Arnold. ${ }^{1}$ Under the restriction of two dimensional flows on the torus $T^{2}=R^{2} /(2 \pi Z)^{2}$ (R: space of real numbers; $Z$ : set of integers), explicit formulas for the commutator, inner product, riemannian connection and geodesic equation are presented. These formulas allow us to calculate the riemannian curvature tensors on the geodesics of any two-dimensional cross-section. By the Jacobi equation in the differential geometry, the stability of the geodesics is determined by the curvatures on them. By the stability it is meant here kinematically how the difference of particle positions between two flows of different initial conditions develops with time. Negative curvature leads to enhanced growth of the deviation in initial conditions with time. (This problem is different from that of the dynamical stability of the velocity field.)

In this paper, we present an explicit form of the geodesic equation for the motion of an ideal fluid on a three-torus $\mathrm{T}^{3}$. It is possible to determine an expression of the riemannian curvature of the group of volume preserving diffeomorphisms. It is interesting to find that the curvature turns out to be non-positive for the twodimensional section consisting of a particular vector (velocity) field (i.e. a simple flow with Beltrami property) and a general vector field.

Consider a flow of an ideal fluid of uniform density in a $n$ dimensional flow domain M ( a bounded riemannian manifold without boundary). The present analysis is aimed at the the flows in three dimensional space although the formulation is valid for $n$ dimensional flows. The manifold $M$ is provided with the metric given by the inner product $(X \cdot Y)$ and the covariant derivative $\nabla_{X} Y$ (or riemannian connection for this metric) at any point $x \in M$ for any two vector fields $X$ and $Y$ on $M$. The covariant 
derivative is a differentiation of the vector field $Y$ in the direction of $X$. When the velocity $v_{t}(y)$ of fluid motion is given for $y \in M$ and $t \in R$, the particle motion $y(t)$ is described by the ordinary differential equation, $d y / d t=v_{t}(y)$ with $y(0)=x \in M$, where $x$ is the initial position of the particle. The solution is written in the form: $y=g_{t}(x)$, which describes a smooth curve in $M$ starting from $x$, usually called Lagrangian particle path.

For each $t$, the mapping $g_{t}: M \rightarrow M$ is an auto-diffeomorphism carrying every particle of the fluid in $M$ from the place it was at time 0 to the place it is at time $t$, and in the other sense for the time parameter $t$ in an open interval $I \subset R, g_{t}$ describes time development of the configuration of particles, called a flow. In case that $v_{t}(x)$ is divergence-free, the diffeomorphism is volume preserving. The volume preserving diffeomorphisms from $M$ to itself form an infinite dimensional group, which is denoted as $\mathcal{D}_{v}(M)$ (shortly $\mathcal{D}_{v}$ ), and the flow $g_{t}$ is a curve in the group $\mathcal{D}_{v}$.

Here we make some remarks about the infinite Lie group $\mathcal{D}_{v}$ according to Ebin \& Marsden. ${ }^{2}$ (i) For two elements $f$ and $g$ in the group, the operation $f g$ is defined by $f \circ g$, i.e. $f \circ g(x)=f(g(x))$ for $x \in M$. Unit element $e$ is the identity map of $M$. Inverse element of $f$ is the inverse map of $f$ itself, so we denote it as $f^{-1}$. (ii) Right translation by an element $g$ is defined by $\mathrm{R}_{g} f=f \circ g$. (iii) The Lie algebra, i.e. the tangent space $\mathrm{T}_{e} \mathcal{D}_{v}$ of the group at the identity $e$, is a set of all divergence-free vector fields on $M$. (iv) Tangent space $\mathrm{T}_{f} \mathcal{D}_{v}$ is a set of all divergence-free vector fields along $f$, i.e. consisting of every vector field $V$ such that $V \circ f^{-1}$ is divergence-free. The right translation of a vector $V_{f} \in T_{f} \mathcal{D}_{v}$ by $g \in \mathcal{D}_{v}$ is defined by $V_{f} \circ g \in T_{f \circ g} \mathcal{D}_{v}$. We use the same notation of the right translation of a vector as that of an element of $\mathcal{D}_{v}$. (v) The initial position $x$ of all the particles is usually taken as the Lagrangian (particle) coordinates. The flow $g_{t}$ starts at e. The fluid velocity with respect to $x$ at each $\mathrm{t}$ is a tangent vector $\dot{g}_{t} \in T_{g_{t}} \mathcal{D}_{v}$. To get the Eulerian velocity field $v_{t}(y)$ at a fixed point $y$ in the space $M$, we must carry this vector to the tangent space at the 
identity $e$. This can be done by the right translation $v_{t}=\dot{g}_{t} \circ g_{t}^{-1}$, or equivalently $\dot{g}_{t}(x)=v_{t}\left(g_{t}(x)\right)$. (vi) The group $\mathcal{D}_{v}$ admits a riemannian metric $<, \quad>$ and riemannian (Levi-Civita) connection $\tilde{\nabla}$.

The metric for two tangent vectors $X, Y \in \mathrm{T}_{f} \mathcal{D}_{v}$ is defined by

$$
<X, Y>\left.\right|_{f}=\left.\int(X(x) \cdot Y(x))\right|_{f(x)} d x
$$

where $d x$ is the (riemannian) volume element on $M$, and $\left.Q\right|_{f}$ denotes the quantity $Q$ evaluated at a point $f$. Due to the volume preserving nature of the mapping, we have the right invariant property: $\left\langle X \circ g, Y \circ g>\left.\right|_{f \circ g}=<X, Y>\left.\right|_{f}\right.$, for $f, g \in \mathcal{D}_{v}$.

Concerning the connection $\tilde{\nabla}_{X} Y$, first we consider right invariant vector fields on $\mathcal{D}_{v}$, i.e. $\tilde{X}(f)=X \circ f, \tilde{Y}(f)=Y \circ f$ for $f \in \mathcal{D}_{v}$ where $X, Y \in T_{e} \mathcal{D}_{v}$ (divergence-free vector fields on $M)$. The connection $\tilde{\nabla}$ determines a new right invariant vector field $\tilde{\nabla}_{X} \tilde{Y}$ on $\mathcal{D}_{v}$ called covariant derivative of $\tilde{Y}$ in the direction of $\tilde{X}$ defined by

$$
\left.\tilde{\nabla}_{\tilde{X}} \tilde{Y}\right|_{f}=\mathrm{P}\left[\nabla_{X} Y\right] \circ f
$$

where $\nabla$ is the riemannian connection of $M$, introduced previously. The covariant derivation $\nabla_{X} Y$ is not necessarily divergence-free for two divergence-free vector fields $X, Y$ on $\mathrm{M}$. The operation $\mathrm{P}[\mathrm{]}$ denotes the projection to divergence-free part on $\mathrm{M}$. It is shown ${ }^{2}$ that the operation (??) gives the right invariant riemannian connection on $\mathcal{D}_{v}$ associated with the riemannian metric (??).

Next we take a curve $g_{t}$ in $\mathcal{D}_{\mathrm{v}}$ which satisfies $\dot{g}_{t} \equiv d g_{t} / d t=v_{t} \circ g_{t}=v_{t}\left(g_{t}\right)$, that is, the flow $g_{t}$ is generated by $v_{t}$. Let $\tilde{X}_{t}$ be a vector field along $g_{t}$ which is given by right translation of a time-dependent vector field $X_{t}$ on $M: \tilde{X}_{t}=X_{t} \circ g_{t}$. Then the connection determines a new vector field called the covariant derivative of $\tilde{X}_{t}$ along $g_{t}$,

$$
\tilde{D} \tilde{X}_{t} / d t=\left(\partial X_{t} / \partial t+\mathrm{P}\left[\nabla_{v_{t}} X_{t}\right]\right) \circ g_{t}
$$

Since $\tilde{D} / d t$ is the differentiation with respect to the parameter $t$, we have to add the term $\partial X_{t} / \partial t$. 
The operations $\{\tilde{X}, \tilde{Y}\} \rightarrow \tilde{\nabla}_{\tilde{X}} \tilde{Y}$ and $\tilde{X}_{t} \rightarrow \tilde{D} \tilde{X}_{t} / d t$ is characterized uniquely by the axioms in the riemannian geometry. ${ }^{3,4}$ In particular, a vector field $\tilde{X}_{t}$ along $g_{t}$ is said to be parallel if the covariant derivative is identically zero. The curve $g_{t}$ is a geodesic when its tangent vector $\dot{g}_{t}$ is parallel along itself (the curve $g_{t}$ ). In $\mathcal{D}_{v}$ endowed with the connection $\tilde{\nabla}$, the geodesics are defined by the equation,

$$
\tilde{D} \dot{g}_{t} / d t=0
$$

The principle of least action asserts that the motion of an ideal fluid is a geodesic with the (weak) riemannian structure (??) and riemannian connection (??). In fact, the action $I$ is defined as

$$
I=\int\left\langle\dot{g}_{t}, \dot{g}_{t}>\left.\right|_{g_{t}} d t=\left.\int d t \int\left(v_{t}(x) \cdot v_{t}(x)\right)\right|_{x} d x,\right.
$$

and the variational problem leads to the equation (??). ${ }^{2}$

Using the right invariant property of the metric $\langle$,$\rangle , and identifying X_{t}$ as $v_{t}$ in (??), we get the following equation,

$$
\tilde{D} \dot{g}_{t} / d t=\left(\partial v_{t} / \partial t+\mathrm{P}\left[\nabla_{v_{t}} v_{t}\right]\right) \circ g_{t}=0 .
$$

For the covariant derivative $\nabla_{v_{t}} v_{t}$, we have the orthogonal decomposition, ${ }^{2}$

$$
\left.\nabla_{v_{t}} v_{t}\right|_{e}=\left.\left\{\mathrm{P}\left[\nabla_{v_{t}} v_{t}\right]-\operatorname{grad} p\right\}\right|_{e}
$$

where the function $p$ is a smooth scalar function on $\mathrm{M}$. Thus, the right translation of equation (??) leads to

$$
\partial v_{t} / \partial t+\mathrm{P}\left[\nabla_{v_{t}} v_{t}\right]=0
$$

or in view of (??), $\partial v_{t} / \partial t+\nabla_{v_{t}} v_{t}=-\operatorname{grad} p$. This is a generalized expression of the Euler equation for an ideal fluid on riemannian manifold $M$ without boundary. It is shown that Eq. (??) holds in most general cases with boundary. ${ }^{2}$ For a flat cartesian space, the covariant derivative reduces to the form

$$
\nabla_{v} v=(\mathrm{v} \cdot \operatorname{grad}) \mathbf{v}
$$


for the velocity field $\mathrm{v}$ with cartesian components $\left(v_{i}\right)$ at $\mathrm{x}=\left(x_{i}\right)$. Thus in terms of the hydrodynamic notations for $n=3$, we have recovered the Euler equation,

$$
\partial \mathbf{v} / \partial t+(\mathbf{v} \cdot \operatorname{grad}) \mathbf{v}=-\operatorname{grad} p, \operatorname{div} \mathbf{v}=0
$$

where $\operatorname{grad}=\left(\partial / \partial x_{i}\right),(i=1,2,3)$ and $p$ is the pressure divided by the uniform fluid density.

In the Lie algebra of the group $\mathcal{D}_{v}$, the commutator $[,]_{*}$ is defined for two divergence-free vector fields $X, Y$ as

$$
[X, Y]_{*}=\tilde{\nabla}_{X} Y-\tilde{\nabla}_{Y} X
$$

The right invariant property of the metric and connection allows us to make corresponding calculation at $e \in \mathcal{D}_{v}$. From now on we write $\tilde{\nabla}$ instead of $\mathrm{P}[\nabla]$.

Given divergence-free vector fields $X, Y, Z$ and $W$, a new divergence-free vector field $\tilde{R}(X, Y) Z$ called the curvature tensor is defined by

$$
\tilde{R}(X, Y) Z=-\tilde{\nabla}_{X} \tilde{\nabla}_{Y} Z+\tilde{\nabla}_{Y} \tilde{\nabla}_{X} Z+\tilde{\nabla}_{[X, Y]} Z
$$

and then the curvature $\tilde{R}_{X Y Z W}$ is given by

$$
\tilde{R}_{X Y Z W}=<\tilde{R}(X, Y) Z, W>
$$

The sectional curvature for the section $\sigma \subset \mathrm{T}_{e} \mathcal{D}_{v}$ spanned by $X$ and $Y$ is

$$
\tilde{K}(\sigma)=<\tilde{R}(X, Y) X, Y>/\left(<X, X><Y, Y>-<X, Y>^{2}\right)
$$

where the denominator is the square of the area of parallelogram spanned by $X$ and $Y$. As we mentioned before, the stability of a geodesic $g_{t}$ is determined by the Jacobi field $A_{t}$ along $g_{t}$. The norm $\left|A_{t}\right| \equiv \sqrt{\left.<A_{t}, A_{t}\right\rangle}$ denotes the evolution of distance between two geodesics $g_{t}^{0}$ and $g_{t}^{s}$ per unit variation of $s$ which start from the same origin $e$ with different initial tangent vectors $X$ and $X+s Y$ respectively: ${ }^{5}$

$$
\left|A_{t}\right|=|Y| t-\frac{1}{6} \tilde{K}(\sigma) t^{3}+O\left(t^{4}\right)
$$


Thus negative $\tilde{K}(\sigma)$ means enhanced deviation of two geodesics in the section $\sigma$ spanned by $X$ and $Y$.

We will now investigate the three-dimensional fluid motion on the flat three-torus $T^{3}=R^{3} /(2 \pi Z)^{3}$, i.e. $x=\left\{\left(x_{1}, x_{2}, x_{3}\right) ; \bmod 2 \pi\right\}$ for $x \in T^{3}$, and the curvature of the group $\mathcal{D}_{v}\left(\mathrm{~T}^{3}\right)$. Note that this $\mathrm{T}^{3}$ is actually bounded manifold without boundary. The elements of the Lie algebra of the group $\mathcal{D}_{v}\left(\mathrm{~T}^{3}\right)$ can be thought of as real periodic vector fields on $T^{3}$ with the divergence-free property. Such a periodic fields are represented by the real part of corresponding complex Fourier forms.

The Fourier base $e^{i \mathbf{k} \cdot \mathbf{x}}$ is denoted by $e_{\mathbf{k}}$, where $\mathbf{k}=\left(k_{i}\right)$ for $i=1,2,3$. We now complexify the Lie algebra, inner product, commutator and the riemannian connection and curvature tensor, so that all these functions become linear (or multilinear) in the complex vector space of the complexified Lie algebra. The functions $e_{\mathbf{k}}\left(\mathbf{k} \in \mathrm{Z}^{3}, \mathbf{k} \neq 0\right)$ form a basis of this vector space. The velocity field is represented as

$$
v_{t}(x) \equiv \mathbf{u}(t, \mathbf{x})=\sum_{\mathbf{k}} \mathbf{u}_{\mathbf{k}}(t) e_{\mathbf{k}},
$$

where $\mathbf{u}(t, \mathbf{x})$ and $\mathbf{u}_{\mathbf{k}}(t)$ have three components, the latter being written as $u^{i}(\mathbf{k})$ too occasionally $(i=1,2,3)$. The Fourier components must satisfy the two properties,

$$
\left(\mathbf{k} \cdot \mathbf{u}_{\mathbf{k}}\right)=0, \quad \mathbf{u}_{-\mathbf{k}}=\mathbf{u}_{\mathbf{k}}^{*}
$$

to represent the solenoidal and real conditions, respectively. Where the asterisk * denotes the complex conjugate. It should be noted that $u_{\mathbf{k}}$ has two independent polarizations consistent with the first condition.

Let us take four vector fields satisfying the conditions (??) : $\mathrm{u}_{\mathrm{k}} e_{\mathrm{k}}, \mathrm{v}_{\mathrm{l}} e_{\mathrm{l}}, \mathrm{w}_{\mathrm{m}} e_{\mathrm{m}}$, $z_{n} e_{n}$. Then we have the following expressions. From (??), the inner product is

$$
<\mathrm{u}_{\mathrm{k}} e_{\mathrm{k}}, \mathrm{v}_{\mathrm{l}} e_{\mathrm{l}}>=(2 \pi)^{3}\left(\mathrm{u}_{\mathrm{k}} \cdot \mathrm{v}_{\mathrm{l}}\right) \delta_{0, \mathrm{k}+\mathrm{l}}
$$

which is non-zero only for $\mathrm{k}+\mathrm{l}=\mathbf{0}$. Using.(??) and operating the projection $\mathrm{P}$, the 
covariant derivative is

$$
\tilde{\nabla}_{\mathbf{u}_{\mathbf{k}} \mathbf{e}_{\mathbf{k}}} \mathbf{v}_{\mathbf{l}} e_{\mathbf{l}}=i\left(\mathbf{u}_{\mathbf{k}} \cdot \mathbf{l}\right) \frac{\mathbf{k}+\mathbf{l}}{|\mathbf{k}+\mathbf{l}|} \times\left(\mathbf{v}_{\mathbf{l}} \times \frac{\mathbf{k}+\mathbf{l}}{|\mathbf{k}+\mathbf{l}|}\right) e_{\mathbf{k}+\mathbf{l}} .
$$

The equation (??) gives the commutator:

$$
\left[\mathbf{u}_{\mathbf{k}} e_{\mathbf{k}}, \mathbf{v}_{\mathbf{l}} e_{\mathbf{l}}\right]_{*}=i\left(\left(\mathbf{u}_{\mathbf{k}} \cdot \mathbf{l}\right) \mathbf{v}_{\mathbf{l}}-\left(\mathbf{v}_{\mathbf{l}} \cdot \mathbf{k}\right) \mathbf{u}_{\mathbf{k}}\right) e_{\mathbf{k}+\mathbf{l}}
$$

From the definitions (??) and (??), the curvature tensor is

$$
\begin{aligned}
& \tilde{R}_{\mathbf{k l m n}}=<\tilde{R}\left(\mathbf{u}_{\mathrm{k}} e_{\mathrm{k}}, \mathrm{v}_{\mathbf{l}} e_{\mathrm{l}}\right) \mathrm{w}_{\mathbf{m}} e_{\mathbf{m}}, \mathbf{z}_{\mathbf{n}} e_{\mathbf{n}}> \\
& \quad=(2 \pi)^{3}\left(\frac{\left(\mathbf{u}_{\mathbf{k}} \cdot \mathbf{m}\right)\left(\mathbf{w}_{\mathbf{m}} \cdot \mathbf{k}\right)}{|\mathbf{k}+\mathbf{m}|} \frac{\left(\mathbf{v}_{\mathbf{l}} \cdot \mathbf{n}\right)\left(\mathbf{z}_{\mathbf{n}} \cdot \mathbf{l}\right)}{|\mathbf{l}+\mathbf{n}|}-\frac{\left(\mathbf{v}_{\mathbf{l}} \cdot \mathbf{m}\right)\left(\mathbf{w}_{\mathbf{m}} \cdot \mathbf{l}\right)}{|\mathbf{l}+\mathbf{m}|} \frac{\left(\mathbf{u}_{\mathbf{k}} \cdot \mathbf{n}\right)\left(\mathbf{z}_{\mathbf{n}} \cdot \mathbf{k}\right)}{|\mathbf{n}+\mathbf{k}|}\right)
\end{aligned}
$$

The curvature $\tilde{R}_{\mathbf{k l m n}}$ takes nonzero value only for $\mathbf{k}+\mathbf{l}+\mathbf{m}+\mathbf{n}=\mathbf{0}$ and nonzero $\mathbf{k}, \mathbf{l}, \mathbf{m}, \mathbf{n}$. (The terms with zero denominator should be deleted.) An analysis for the distance of each particle convected by two different velocity fields of $T^{2}$, which is related to the Jacobi field (??), has been carried out by Hattori ${ }^{6}$ and found to be consistent with (??). This analysis can be extended to $\mathrm{T}^{3}$ without difficulty.

The geodesic equation (??) reduces to

$$
\sum_{\mathbf{k}} \frac{\partial u^{m}(\mathbf{k})}{\partial t}+i \sum_{\mathbf{p} \nmid \mathbf{q}=\mathbf{k}} \sum_{j, l} k_{j}\left(\delta_{m l}-\frac{k_{m} k_{l}}{k^{2}}\right) u^{j}(\mathbf{p}) u^{l}(\mathbf{q})=0
$$

by using (??) where $\delta_{i j}$ is Kronecker's delta. It is interesting to find that this is exactly identical to the Fourier representations of the Euler equation, or the NavierStokes equation when the viscosity coefficient $\nu$ vanishes. ${ }^{7}$

In two-dimensional case, we may take $\mathbf{k}=\left(k_{1}, k_{2}, 0\right)$ and $\mathbf{u}_{\mathbf{k}}=i\left(k_{2},-k_{1}, 0\right)$, and similar expressions for the other vectors. Then it can be readily shown that our formulas reduce to those of Arnold. ${ }^{1}$

As an application, we consider a flow with Beltrami property, that is, we assume that the velocity field $\mathbf{U}_{\mathbf{p}}=\mathbf{u}_{\mathbf{p}} e_{\mathbf{p}}+\mathbf{u}_{-\mathbf{p}} e_{-\mathbf{p}}$, i.e. $2 \operatorname{Re}\left[\mathbf{u}_{\mathbf{p}} e^{i \mathbf{p} \cdot \mathbf{x}}\right]$, satisfies the condition, 
curl $\mathbf{U}_{\mathbf{p}}=\lambda \mathbf{U}_{\mathbf{p}}$ for a parameter $\lambda \in \mathrm{R}$. This eigenvalue problem can be solved with $\lambda^{2}=|\mathbf{p}|^{2}$. It is readily shown that $\mathbf{U}_{\mathbf{p}}$ is a steady-state solution. Let $\mathbf{X}=\sum \mathbf{v}_{\mathbf{l}} e_{\mathbf{l}}$ be any velocity field satisfying (??). Using the above formulas, we obtain

$$
<\tilde{R}\left(\mathbf{U}_{\mathbf{p}}, \mathbf{X}\right) \mathbf{U}_{\mathbf{p}}, \mathbf{X}>=-(2 \pi)^{3} \sum_{\mathbf{l}} \frac{\left|\left(\mathbf{u}_{\mathbf{p}} \cdot \mathbf{l}\right)\right|^{2}}{|\mathbf{p}+\mathbf{l}|^{2}}\left|\frac{\left(\mathbf{u}_{\mathbf{p}} \cdot \mathbf{l}\right)^{2}}{\left|\left(\mathbf{u}_{\mathbf{p}} \cdot \mathrm{l}\right)\right|^{2}}\left(\mathbf{v}_{\mathbf{l}} \cdot \mathbf{p}\right)-\left(\mathbf{v}_{\mathbf{l}+\mathbf{2}} \cdot \mathbf{p}\right)\right|^{2} \leq 0 .
$$

This non-positive property is considered to be a three dimensional version of the Arnold's result for the curvature of the group $\mathcal{D}_{v}\left(\mathrm{~T}^{2}\right)$ in any two-dimensional section containing the direction $\xi$ represented by the stream function $\frac{1}{2}\left(e_{\mathbf{p}}+e_{-\mathbf{p}}\right)$.

The above result can be extended to a two-mode Beltrami flow $\mathbf{U}_{\mathbf{p}, \mathbf{q}}$ which is defined by the linear combination $\mathbf{U}_{\mathbf{p}}+\mathbf{U}_{\mathbf{q}}$ of two Beltrami flows $\mathbf{U}_{\mathbf{p}}$ and $\mathbf{U}_{\mathbf{q}}$. Although it is not difficult to derive the curvature formula in the section of $\mathbf{U}_{\mathbf{p}, \mathbf{q}}$ and the general direction $\mathbf{X}$, we show here only the case in which $\mathbf{X}$ is another Beltrami flow represented by $\mathbf{V}_{\mathbf{p}, \mathbf{q}}=\mathbf{V}_{\mathbf{p}}+\mathbf{V}_{\mathbf{q}}$ where $\mathbf{V}_{\mathbf{p}}=\mathbf{v}_{\mathbf{p}} e_{\mathbf{p}}+\mathbf{v}_{-\mathbf{p}} e_{-\mathbf{p}}$. The two polarization amplitudes $v_{\mathbf{p}}$ and $\mathbf{u}_{\mathbf{p}}$ are linearly independent and the Beltrami condition is satisfied with $\lambda=|\mathbf{p}|$. Then the sectional curvature is

$$
\begin{aligned}
<\tilde{R} & \left(\mathbf{U}_{\mathbf{p}, \mathbf{q}}, \mathbf{V}_{\mathbf{p}, \mathbf{q}}\right) \mathbf{U}_{\mathbf{p}, \mathbf{q}}, \mathbf{V}_{\mathbf{p}, \mathbf{q}}>/(2 \pi)^{3} \\
= & -N_{+}\left|\left(\mathbf{u}_{\mathbf{p}} \cdot \mathbf{q}\right)\left(\mathbf{v}_{\mathbf{q}}^{*} \cdot \mathbf{p}\right)-\left(\mathbf{u}_{\mathbf{q}}^{*} \cdot \mathbf{p}\right)\left(\mathbf{v}_{\mathbf{p}} \cdot \mathbf{q}\right)\right|^{2} \\
& -4 N_{+} \operatorname{Im}\left[\left(\mathbf{u}_{\mathbf{p}} \cdot \mathbf{q}\right)\left(\mathbf{v}_{\mathbf{p}}^{*} \cdot \mathbf{q}\right)\right] \cdot \operatorname{Im}\left[\left(\mathbf{u}_{\mathbf{q}} \cdot \mathbf{p}\right)\left(\mathbf{v}_{\mathbf{q}}^{*} \cdot \mathbf{p}\right)\right] \\
& -N_{-}\left|\left(\mathbf{u}_{\mathbf{p}} \cdot \mathbf{q}\right)\left(\mathbf{v}_{\mathbf{q}} \cdot \mathbf{p}\right)-\left(\mathbf{u}_{\mathbf{q}} \cdot \mathbf{p}\right)\left(\mathbf{v}_{\mathbf{p}} \cdot \mathbf{q}\right)\right|^{2} \\
& -4 N_{-} \operatorname{Im}\left[\left(\mathbf{u}_{\mathbf{p}} \cdot \mathbf{q}\right)\left(\mathbf{v}_{\mathbf{p}}^{*} \cdot \mathbf{q}\right)\right] \cdot \operatorname{Im}\left[\left(\mathbf{u}_{\mathbf{q}}^{*} \cdot \mathbf{p}\right)\left(\mathbf{v}_{\mathbf{q}} \cdot \mathbf{p}\right)\right]
\end{aligned}
$$

where $N_{ \pm}=2 /|\mathbf{p} \pm \mathbf{q}|^{2}$ 
Next we show an interesting example, that is an application to the $\mathrm{ABC}$ flow ${ }^{8}$ represented by

$$
\begin{aligned}
\mathrm{U}_{\mathrm{ABC}}= & \mathrm{A}\left[(i, 1,0) e^{i x_{3}}+(-i, 1,0) e^{-i x_{3}}\right] \\
& +\mathrm{B}\left[(0, i, 1) e^{i x_{1}}+(0,-i, 1) e^{-i x_{1}}\right] \\
& +\mathrm{C}\left[(1,0, i) e^{i x_{2}}+(1,0,-i) e^{-i x_{2}}\right]
\end{aligned}
$$

where $\mathrm{A}, \mathrm{B}, \mathrm{C} \in \mathrm{R}$. This is a three-mode Beltrami flow, i.e. each term on the right hand side satisfies the Beltrami property with $\lambda=-1$. We have another $\mathrm{ABC}$ flow $\mathrm{U}_{\mathrm{A}^{\prime} \mathrm{B}^{\prime} \mathrm{C}^{\prime}}$ for $\left(\mathrm{A}^{\prime} ; \mathrm{B}^{\prime}, \mathrm{C}^{\prime}\right) \neq(\mathrm{A}, \mathrm{B}, \mathrm{C})$. It is straightforward to show that

$$
\begin{aligned}
& <\tilde{R}\left(\mathbf{U}_{\mathrm{ABC}}, \mathrm{U}_{\mathrm{A}^{\prime} \mathrm{B}^{\prime} \mathrm{C}^{\prime}}\right) \mathbf{U}_{\mathrm{ABC}}, \mathbf{U}_{\mathrm{A}^{\prime} \mathrm{B}^{\prime} \mathrm{C}^{\prime}}> \\
& \quad=-2\left\{\left(\mathrm{AB}^{\prime}-\mathrm{BA}^{\prime}\right)^{2}+\left(\mathrm{BC}^{\prime}-\mathrm{CB}^{\prime}\right)^{2}+\left(\mathrm{CA}^{\prime}-\mathrm{AC}^{\prime}\right)^{2}\right\}
\end{aligned}
$$

namely the curvature is non-positive. In particular the curvature vanishes when $A / A^{\prime}=B / B^{\prime}=C / C^{\prime}$. It is interesting to find that even in the case where both $(\mathrm{A}, \mathrm{B}, \mathrm{C})$ and $\left(\mathrm{A}^{\prime}, \mathrm{B}^{\prime}, \mathrm{C}^{\prime}\right)$ are close and are not in the domain of chaos, ${ }^{8}$ particle motion by $U_{A^{\prime} B^{\prime} C^{\prime}}$ will not be predicted from the particle motion by $U_{A B C}$ in the course of time.

It is expected that the present method may be applied to the analysis of rate of growth of line-element or surface-element in various flow fields and turbulence. 


\section{References}

1 V. I. Arnold, Ann. Inst. Fourier, Grenoble 16, 1, 319 (1966); Mathematical Methods of Classical Mechanics, Appendix 2 ( Springer-Verlag, New York, 1978).

${ }^{2}$ D. G. Ebin and J. Marsden, Ann. Math. 90, 102 (1970).

3 J. Milnor, Morse Theory (Princeton University, New Jersey, 1963).

${ }^{4}$ S. Kobayashi and K. Nomizu, Foundations of Differential Geometry Vol. 1 (John Wiley \& Sons, New York, 1969)

5 N. J. Hicks, Notes on Differential Geometry (Van Nostrand, Princeton, N.J., 1965)

6 Y. Hattori, Master thesis, Dept. of Phys., Univ. of Tokyo (1990).

7 R. Kraichnan, J. Fluid Mech. 5, 497 (1959).

8 T. Dombre, U. Frisch, J. M. Greene, M. Hénon, A. Mehr, \& A. M. Soward,

J. Fluid Mech. 167, 353 (1986). 